\title{
1 The psychological and social consequences of single-sided deafness in
}

\section{2 adulthood}

4 Laura Lucas ${ }^{1,2}$, Roulla Katiri ${ }^{3}$, and Pádraig Thomas Kitterick ${ }^{1,2}$

$5{ }^{1}$ National Institute for Health Research Nottingham Biomedical Research Centre, Ropewalk House,

6 Nottingham NG1 5DU, United Kingdom

$7 \quad{ }^{2}$ Otology and Hearing group, Division of Clinical Neuroscience, School of Medicine, University of

8 Nottingham, Nottingham NG7 2UH, United Kingdom

$9 \quad{ }^{3}$ Audiology Department, Mater Misericordiae University Hospital, Dublin 7, Ireland

11 Address correspondence to:

12 Dr Padraig Kitterick

13 National Institute for Health Research Nottingham Biomedical Research Centre

14 Ropewalk House, 113 The Ropewalk

15 Nottingham, NG1 5DU, United Kingdom

16 Telephone: 00441158232626

Fax: 00441158232615

17 Email: Padraig.Kitterick@nottingham.ac.uk

18 Conflicts of Interest and Source of Funding:

19 This work was supported by infrastructure funding from the National Institute for Health Research

20 (NIHR). The views expressed are those of the author(s) and not necessarily those of the NHS, the

21 NIHR or the Department of Health.

23 This is an Accepted Manuscript of an article published by Taylor \& Francis in the

24 International Journal of Audiology and available online at: http://www.tandfonline.com/toc/iija20/ 


\section{Abstract}

27 Objectives: This study examined the subjective psychological and social effects of highly 28 asymmetric hearing loss (single-sided deafness) in adults.

29 Design: Three group interviews were conducted using the critical incidence technique and analysed using an inductive thematic analysis.

31 Study sample: Eight adults with a clinical diagnosis of a moderately-severe hearing loss or

32 greater in one ear and normal or near-normal hearing in the other ear.

33 Results: A range of functional hearing difficulties associated with single-sided deafness including impaired speech in background noise and reduced spatial awareness were reported to affect social and psychological well-being. Social consequences of single-sided deafness resulted from activity limitations and participation restrictions including withdrawal from and within situations. Participants reported psychological effects including worrying about losing the hearing in their other ear, embarrassment related to the social stigma attached to hearing loss, and reduced confidence and belief in their abilities to participate.

Conclusions: Single-sided deafness can be associated with many negative consequences.

41 Counselling may help overcome the psychological consequences of hearing loss regardless of whether technological support such as a hearing aid is prescribed. The audiological management of these individuals should support the development of listening strategies and set appropriate expectations for participation in everyday listening situations.

Key Words: unilateral hearing loss; unilateral deafness; single-sided deafness; SSD; hearing

47 function; listening difficulties; psychosocial impact; critical incidence technique 


\section{Introduction}

Single-sided deafness (SSD) refers to a highly asymmetric unilateral hearing loss. An international consensus statement has defined SSD as a 'severe-to-profound' hearing loss in one ear (pure-tone average threshold $\geq 60 \mathrm{~dB} \mathrm{HL}$ ) and normal or near-normal hearing in the other ear (pure-tone average threshold $<30 \mathrm{~dB}$ HL) (Vincent et al, 2015). The prevalence of SSD has been estimated to be about $1 \%$ of the general population (Davis, 1995). SSD is often associated with sudden onset sensorineural hearing loss, the incidence of which is approximately 20 cases per 100,000 inhabitants per year in developed countries (Byl, 1984). The incidence of SSD in the United Kingdom has been estimated to be between 7000-9000 new cases per year based on available data (Baguley et al, 2006).

While access to one well-functioning ear allows individuals with SSD to appear largely unimpaired in the confines of a quiet room, their lack of access to two functioning ears can lead to a clinically-significant degree of audiological disability in everyday life (ChoissoineKerdel et al, 2000; Dwyer et al, 2014; Iwasaki et al, 2013; Newman et al, 1997). The loss of hearing in one ear severely disrupts the spatial aspects of hearing (Douglas et al, 2007) and impairs the ability to understand speech in the presence of background noise (Hawley et al, 2004; Welsh et al, 2004). It can be difficult to restore access to sound in the impaired ear through amplification using conventional devices such as a highpowered acoustic hearing aid due to the sensorineural nature and extent of the hearing loss in these individuals (Valente et al, 2015). The current standard of care for SSD in the United Kingdom is the contralateral routing of signals (CROS) aid (Kitterick et al, 2014), a device which reroutes sound from the side of the impaired ear to the hearing ear for the benefit of speech understanding in noise (Arndt et al, 2011; Arndt et al, 2017; 
74 Busk et al, 2014; Hol et al, 2010; Lin et al, 2006; Niparko et al, 2003; Ryu et al, 2015).

75 A similar effect can be achieved using a bone-conduction hearing device (BCHD)

76 (Desmet et al, 2012; Finbow et al, 2015; Kompis et al, 2017; Newman et al, 2010; Saliba et al, 2011; Schrøder et al, 2010; Wesarg et al, 2013) with the potential for even better benefits to speech perception and sound quality compared to CROS devices (Kitterick et al, 2016). Cochlear implantation has the additional benefits of restoring access to binaural cues that underpin speech perception in noise and sound localisation (Arndt et al, 2011; Arndt et al, 2017; Finke et al, 2017b; Hassepass et al, 2016; Jacob et al, 2011; Mertens et al, 2015; Távora-Vieira, 2015; Vermiere and Van de Heyning, 2009), which longitudinal studies have shown to be sustained over the long term ( $>10$ years) (Arndt et al, 2017).

The functional consequences of SSD have been associated with a range of psychological and social consequences. For example, individuals with SSD can report increased stress levels related to their need to seek out optimal positions within social settings in order to maximise their ability to hear and participate (Hansson, 1993, cited in Wie et al, 2010). As a result, individuals with SSD can report feeling excluded from social situations (Wie et al, 2010) and perceive their social life to be restricted by their hearing loss (Subramanium et al, 2005), leading to high levels of hearing handicap (Dwyer et al, 2014; Iwasaki et al, 2013). Numerous studies have demonstrated that rerouting sounds between the ears using CROS or BCHD significantly reduces the perceived difficulties with listening in everyday life thereby improving hearing-specific quality of life (Busk et al, 2014; Finbow et al, 2015; Hol et al, 2010; Kompis et al, 2017; Lin et al, 2006; Niparko et al, 2003; Ryu et al, 2015; Schrøder et al, 2010; Wazen et al, 2003). However, some studies have suggested that there can be increased aversion to loud sounds with the 
use of CROS devices (Lin et al, 2006). Cochlear implantation has also been associated with benefits to hearing-specific quality of life, with the additional benefit of reduced difficulty with identifying the location of sound sources, navigating everyday environments and tinnitus relief (Arndt et al, 2011; Arndt et al, 2017; Finke et al, 2017a; Härkönen et al, 2015; Mertens et al, 2015; Ramos et al, 2015; Távora-Vieira et al, 2015; Van de Heyning et al, 2008). Arndt et al (2011) also demonstrated that cochlear implantation can have broader benefits on health related quality of life as measured by the Health Utilities Index Mark 3. However, cost-benefit analyses are still needed to assess whether the size of these broader benefits are sufficient to justify the costs of cochlear implantation (Kitterick et al., 2015). These analyses will need to be conducted from a specific perspective as costs will vary across countries, healthcare systems, and funding models (private versus publicly funded).

Previous studies have primarily used self-report questionnaire instruments to both characterise the burden that SSD places on the individuals and the benefits that devices can have on quality of life. Examples include the Monaural Auditory Capacity Assessment Scale (MACAS) (McLeod et al, 2008), the Speech Spatial and Qualities of Hearing Scale (SSQ) (Gatehouse \& Noble, 2004), and the Auditory Profile of Hearing Aid Benefit (APHAB) (Cox and Alexander, 1995). While these instruments are efficient to administer and straightforward to analyse, it is possible that the use of closed questions places limits on the breadth and depth of the information gathered (McColl et al, 2001; O'Cathain \& Thomas, 2004).

Qualitative methodologies can be particularly powerful when seeking to capture the full range of difficulties and consequences associated with a health condition (Flanagan, 1954).

Very few studies have applied qualitative methods to explore the consequences of SSD.

Giolas \& Wark (1967) use a method called the Critical Incident Technique (CIT) to generate 
124 discussions around the situations that patients themselves recalled as being problematic as

125 a result of their hearing loss. The results helped enumerate the many ways in which SSD

126 imposes functional limitations on the individual, largely through impairments to speech

127 understanding in noise and the ability to localise sounds. However, to date the psychological

128 and social consequences that SSD imposes have not been explored or characterised in the

129 same level of detail that qualitative methodologies can provide.

130

131 The aim of the present study was to address this gap in the literature by conducting an in-

132 depth qualitative investigation of the psychological and social consequences of SSD on

133 everyday life. As there may be distinct differences between short and longer-term

134 consequences of unilateral hearing loss, particularly where its onset is sudden (Sano, 2013),

135 the current study focussed on the longer-term consequences in those who had lived with SSD

136 for at least a year. By using the CIT method, the study was able to explore the full extent of

137 these consequences and provide insight into both the situations in which they arise and the

138 impacts they have on the individual. The study also gathered information on the strategies

139 that are developed to cope with these consequences and to enable continued participation in

140 everyday life. The results increase our understanding of the overall health and well-being of

141 adults with SSD.

142

143 Materials and Methods

144 Sampling and recruitment

145 Participants had a clinical diagnosis of a sensorineural highly asymmetric unilateral hearing

146 loss (single-sided deafness) for a period of at least 12 months, defined as a pure-tone average

147 of $\geq 60 \mathrm{~dB}$ HL with at least two thresholds $\geq 65 \mathrm{~dB} \mathrm{HL}$ at 1,2 , or $4 \mathrm{kHz}$ in one ear and an 
148 average threshold of $\leq 30 \mathrm{~dB}$ HL in their other ear. Participants had to confirm by self-report

149 that their hearing loss had not changed in severity within the last 12 months.

151 Participants were recruited through the NIHR Nottingham Hearing Biomedical Research Unit

152 participant database via email or postal invitation specifically for the purpose of this study.

153 Advertisements were also placed in the Audiology services and Ear, Nose and Throat clinics

154 in local hospitals, the mailing lists of hearing-related charities, and on social media websites.

155 No restrictions were placed on a participants' age, gender or employment status. Participants

156 had not already taken part in any related research studies. Participants were screened using an

157 online questionnaire to confirm their eligibility for the study. Those who passed the screening

158 phase were asked to provide a copy of a recent audiogram (taken within the past two years)

159 or invited to take part in a short in-person audiometry assessment to confirm their eligibility.

160 The assessment consisted of otoscopy followed by threshold measurements for each ear using

161 air-conduction audiometry, both conducted in accordance with the British Society of

162 Audiology recommended procedures (BSA 2010, 2012).

163

\section{Participants}

165 Eight adults with single-sided deafness living in the UK participated in the study (Table 1).

166 All participants were native speakers of English. Participants' aetiologies were mixed and

167 included vestibular schwannoma, meningitis, and idiopathic hearing loss. The mean age was

16857 years (range $=37-71)$ and the average duration of hearing loss was 13 years (range $=13$

169 months-28 years). The mean threshold in the better ear was $13 \mathrm{~dB}$ HL (range $=4-30 \mathrm{~dB} \mathrm{HL}$ )

170 and $82 \mathrm{~dB}$ HL in the other ear (range=61-119 dB HL). A majority of participants (7 out of 8)

171 reported experiencing tinnitus with varying levels of severity. 


\section{Procedure}

174 Three group interviews were conducted using the Critical Incident Technique (CIT). The CIT

175 was employed to create a semi-structured discussion (Flanagan, 1954) whereby participants

176 themselves generate all topics for discussion without prior discussion with others or the

177 researchers facilitating the interview. The rationale for the use of this approach was to ensure,

178 as far as practically possible, that the choice of topics for discussion was not unduly

179 influenced by the prior expectations of the facilitators or only a small vocal minority, but

180 rather reflected the issues that were seen as important and relevant to individual participants

181 (Glaser \& Strauss, 1967). Table 2 describes the specific sequence of activities that were

182 undertaken in all three interviews.

183

184 Participants were allocated to the interviews according to the order in which they were recruited. Two participants took part in each of the first two interviews, the aim of which was to gather in-depth information on the experiences of adults with single-sided deafness. A

187 third interview was conducted with four participants to: (a) provide further insight into where

188 those experiences converged or diverged between individuals, who differed in their age,

189 gender and aetiologies; and (b) assess whether data saturation had been reached (Fusch \&

190 Ness, 2015). The study protocol specified that interim analyses would be conducted after the

191 second and subsequent interviews, and that additional interviews would only be conducted if

192 new topics were still likely to be identified.

193

194 All participants provided written informed consent prior to participation and audio recordings

195 of all discussions were made for written transcription. Two facilitators were present at each

196 interview. A primary facilitator (moderator) was responsible for maintaining the discussion

197 between participants and ensuring all participants contributed to each discussion. A co- 
198 facilitator sought clarification where the audio recording may not have been clear and

199 documented interactions, contextual cues and nonverbal behaviors amongst participants. The

200 discussion was allowed to continue as long as the participants were willing or until all the

201 topics had been exhausted, whichever came first. The research was approved by Central

202 Bristol NHS Research Ethics Committee (REC number: 14/SW/1065) and sponsored by

203 Nottingham University Hospitals NHS Trust.

204

205 Data Analysis

206 Audio recordings of the interviews and the focus group were transcribed verbatim. A

207 thematic analysis was conducted using NVivo 10 (QSR International, Melbourne, Australia).

208 Themes were identified inductively after assigning codes to the transcripts (Braun \& Clarke,

209 2006). Each researcher first read the entire dataset and assigned each individual piece of

210 information one or more thematic codes. The codes were chosen to factually describe the

211 nature of the information being discussed in as much detail as possible and were initially

212 generated by each researcher independently based on their interpretation of the transcripts.

213 After an initial review of the transcripts, the choice of codes used by each researcher was

214 discussed and any disagreements resolved by consensus. The transcripts were then recoded

215 by both researchers using the revised code set. Codes that referred to similar or associated

216 information were grouped into principal themes and sub-themes by the two researchers.

217 Disagreements were resolved by consensus.

219 Results

220 A total of 102 topics were submitted by participants across the three interviews that identified 22177 situations in which participants experienced difficulty because of their hearing loss and 25 
222 situations in which participants either did not experience difficulty or experienced positive consequences (see Table, Supplemental Digital Material 1). An interim analysis of the topics of discussion between interview one and two identified a high degree of consistency with similar topics emerging in both interviews (Table 3). Although the larger sample size of the third interview may have limited the depth of the discussion relative to the two earlier interviews, the topics raised by this larger group were consistent with the first two interviews. A further interim analysis after the third interview showed that no new topics emerged consistently, suggesting that data saturation had been reached (Fusch \& Ness, 2015). Following the study protocol, no further interviews were conducted.

The following sections describe four distinct but related overarching themes in the data related to single-sided deafness: (1) the functional consequences; (2) the psychological consequences; (3) the social consequences; and (4) the positive consequences. The analysis also identified coping strategies that individuals with single-sided deafness develop and employ to maintain social function. The main subthemes are indicated through the use of

237 italic font in the following sections.

\section{Functional Consequences}

240 Difficulties with recognising and understanding speech were reported by all participants.

241 Quiet situations presented the least difficulties as long as only one person is talking at any

242 time and the talker is in view. In both quiet and noisy situations, participants recognised that

243 it was important for the sound of interest to be on the side of their better ear to maximise speech understanding. Noisy situations presented greater difficulties for the participants, particularly where background noise includes speech or is dynamic in nature. P3 said, "If 
247 noise”. An associated increase in listening effort lead to high levels of fatigue, particularly in

248 situations where participants were unable to move to a more favourable listening position.

249 Participants also described how turning their head to ensure that the sound source is on their

250 non-impaired side could be tiring and uncomfortable. Participants reported that their speech

251 perception abilities are also hindered by the acoustics of a setting. Wide-open spaces

252 furnished with hard surfaces that reflect sound and produce echoes (examples included train

253 stations and airports) were particularly problematic.

254

255 Without the benefits of binaural hearing, participants reported difficulty with sound

256 localisation and the ability to selectively attend to one sound, which to some were deemed

257 the most inconvenient implications of their hearing loss. The inability to localise sounds was

258 reported to cause difficulties when identifying the direction of unexpected sounds, with

259 participants reporting that they often presumed (incorrectly) that all sound sources are located

260 on the side of their non-impaired ear. P1 talked about her first experience of trying to cross a

261 road after losing her hearing due to meningitis: "I didn't know where the traffic was, it just

262 seemed to be all around and that was quite scary". A lack of access to binaural cues also

263 meant that participants were unable to benefit from 'surround sound' in the cinema, or had

264 difficulties when listening to music or audiobooks that exploit stereo effects, rendering some

265 sounds inaudible when listening over headphones. Participants also described a heightened

266 awareness of sounds which was most apparent when they first lost their hearing. P6 said

267 "When I first walked out into the street and buses were going past it was scary...you're so

268 super aware of every single noise”.

269

270

Psychological consequences 
271 Participants reported that their hearing loss had many effects on both their mental and

272 emotional wellbeing. The initial reaction to their hearing loss differed depending upon

273 whether the loss of hearing was sudden or whether it had a determined cause. Participants

274 who had experienced a sudden loss reported feelings of 'shock' and 'fear'. P6 described the

275 experience as "devastating" and "life-changing", especially upon realising his hearing loss

276 was permanent. The psychological impact for those whom the cause of their hearing loss had

277 been determined appeared to be less severe. P5, who lost his hearing after surgery to remove

278 a vestibular schwannoma, said: "It kind of feels like although the single-sided hearing has

279 changed my life, so much else happened...I knew I was going to lose my hearing so I'd

280 almost prepared myself for it amongst other things". Some participants found it hard to

281 accept their hearing loss and linked it to feelings of depression and anxiety. P7 said " $I$

282 remember at one stage, a few weeks after it all happened, just sitting in absolute despair

283 saying I just cannot live for the rest of my life like this". Those who had experienced a sudden

284 hearing loss experienced heightened anxiety and worry about losing the hearing in their

285 other ear, whilst all participants were concerned about age-related decline in their remaining

286 'good' ear.

287

288 Participants felt that there was a 'social stigma' or negative perception about their hearing

289 loss from others who often had a lack of understanding and empathy for their communication

290 difficulties. They reported often being wrongly perceived as being rude or antisocial if they

291 failed to respond to someone calling their name or saying 'excuse me'. P1 described the

292 reactions of a stranger when she did not hear them approaching on a bicycle: "I was walking

293 on a path at a nature reserve recently and a cyclist came up on my deaf side and rang his

294 bell. I didn't hear him. Then he overtook and said "are you deaf or something?”. Many

295 participants explained how it took time to accept their hearing loss and admit it to others. P4 
said, "It did take me a long time to say [I'm deaf] to people. I felt embarrassed. I didn't want

to admit it or something. But now I just say 'look I'm deaf in that ear'”.

298

299 Participants also reported feelings of self-stigma (negative perception of oneself due to

300 hearing loss) and low self-efficacy (belief in ones' ability to participate). Participants reported

301 often feeling 'stupid' or 'embarrassed' due to their communication difficulties, particularly

302 when they had misheard what someone had said or had wrongly identified who was talking in

303 a group of people. Some felt like a hindrance as they had to rely on other people to be

304 involved in a conversation, or felt 'guilty' if they had missed what someone had said to them.

305 Although participants recognised that their hearing loss had reduced their ability to cope with

306 many everyday situations, they did not appear to consider themselves to be in poor health

307 because of their hearing loss. 'Health' for these individuals was more commonly associated

308 with pain and chronic illness.

\section{Social consequences}

311 Participants reported problems with social interactions. Interactions with strangers were

312 considered more difficult than interactions with familiar people (i.e. family and friends).

313 Strangers showed a lack of awareness and understanding about effective strategies for

314 communicating with someone who has a hearing loss, often shouting rather than

315 repositioning themselves on the side of their 'good' ear. Familiar voices were reported as less

316 difficult to comprehend than unfamiliar voices and those with poor enunciation. Family and

317 friends were seen as more understanding and empathetic and were relied upon to provide

318 support in challenging listening situations. However, even the closest relatives and friends

319 could be resistant to adjusting their behaviours and could show frustration when asked to

320 repeat themselves or talk more slowly. Participants reported a reduction in the amount of time 
321 they spent communicating with their spouse due to their hearing loss. They also reported a

322 lack of conversational intimacy as they did not always feel able to engage in conversations at

323 quiet levels without risk of embarrassment. Participants reported feeling marginalised at

324 social events, especially those involving younger family members who are often unaware of

325 the difficulties that hearing loss creates.

327 Some participants reported that their hearing loss had effects on social interactions in the workplace. Colleagues would often not be mindful of their hearing difficulties and would talk quietly or position themselves on the side of their impaired ear. Participants also worried that they would be perceived as less capable of conducting their job. This concern was particularly acute among those who worked in a noisy office environment where successful communication with others was a key element of their role. P4 felt that the difficulties she had experienced due to her hearing loss eventually contributed to her leaving her profession as a journalist.

Participants recognised that their willingness to interact with others and participate in social situations had changed since their hearing loss leading them to withdraw from situations or withdraw within situations. Some participants no longer enjoyed being in places with lots of background noise as it left them feeling frustrated and isolated. As a result, these individuals preferred to avoid or withdraw from participating in such situations which could lead to reduced self-esteem. P3 said "If I can get out of going to an event I do, because I wouldn't be able to enjoy the conversation... I wouldn't be able to hear properly". Participants also reported withdrawing from meetings at work where they anticipated that it would be difficult to participate. Other participants described how they felt that it was important to engage back into their everyday life, especially by having a physical presence at social events they 
346 enjoyed attending. However, in more challenging situations, such as those with background

347 noise or where they were unable to choose a favourable positioning in a room, they would

348 often withdraw within themselves as they were not able to wholly engage and participate. In

349 turn, this could lead individuals to feel detached from the social group.

\section{$351 \quad$ Positive effects}

352 The limited positive effects identified by participants primarily encompassed a positive effect on the quality of their sleep as lying with their good ear on the pillow blocked out unwanted background noise. Conversely, some participants worried about failing to hear important sounds such as a smoke alarm, telephone ringing or a child crying whilst they were sleeping. All participants reported that they had been able to find constructive ways in which to adapt to their hearing loss and recognised the importance of positive support from family and friends. These individuals acted as communication partners by relaying important information which may otherwise be missed in conversation. Several participants also reported that the hearing in their 'good' ear appeared enhanced since the onset of their

361 hearing loss.

\section{Coping strategies}

364 Coping strategies were developed by participants to aid engagement and participation in 365 everyday situations. These strategies were primarily a result of self-learning and adaptation due to a lack of formal information and advice from clinicians. Their use of coping strategies appeared to be dependent upon factors including personality traits, social and professional circumstances, and personal attitudes towards hearing loss. Positioning in a social setting was regarded as highly important for participants to maximise the signal-to-noise ratio at their 
370 better ear and to access visual cues (e.g. lip-reading) to aid their ability to understand speech.

371 Participants also highlighted the importance of support from family and friends both in

372 communicating in challenging listening situations and to help in potentially-dangerous

373 situations such as crossing a road when they are unable to localise the sound of traffic or

374 identify the distance of vehicles.

375

376 Participants reported that they do not generally wear hearing-assistive devices. In general,

377 they did not find conventional hearing aids to be beneficial for their hearing. Consequently,

378 they felt frustrated that their hearing loss was an invisible condition and some had even

379 considered wearing a hearing aid solely to remind others of their hearing loss. Those who had

380 trialled a contralateral routing of signals (CROS) hearing aid (Harford and Barry 1965)

381 deemed its benefits to be limited and did not persist with its use or use it regularly. Some

382 participants relied on other technologies to enhance their hearing and listening abilities such

383 as using subtitles, a speakerphone, a sound-bar for the television, or headphones that can be

384 manipulated so that all sounds play in one-ear only.

386 Discussion

387 The primary aim of the current study was to explore the lasting psychological and social

388 consequences that SSD places on the individual using a technique that attempts to isolate the

389 researchers from the process of selecting topics for discussion (Glaser \& Strauss, 1967). It is

390 inevitable that there will be individual differences in how people cope with a health condition

391 such as SSD and the degree to which the condition imposes burden upon them. However,

392 consistency was observed both in the topics that were identified across the interviews (Table

393 3) and the content of the subsequent discussions, as indicated by the well-defined themes and 
394 sub-themes that emerged in the inductive thematic analysis. As only a small number of

395 patients with acquired SSD were consulted in this study, caution should be exercised in

396 generalising the results to the broader population of patients with SSD that encompasses a

397 greater variation in aetiology and duration of deafness than sampled in the present study and

398 also includes those deafened congenitally. However, it is notable that the findings form

399 strong parallels with those of previous studies whose samples differed from that of the current

400 study on some of these factors (e.g. Giolas \& Wark, 1967; Sano, 2013; Subramanium et al,

401 2005; Wie et al, 2010). This apparent consistency prompts the conclusion that there are

402 common longer-term psychological and social consequences of SSD that arise among

403 individuals despite differences in their age, aetiology, and duration of monaural auditory

404 deprivation. The current thematic analysis identified recurring consequences that included

405 worry about losing the hearing in the better ear, strong negative emotions including

406 embarrassment and frustration, low self-efficacy, and negative coping strategies such as

407 withdrawing from and within challenging everyday listening situations.

408

409 While one might expect that the effects of SSD would be exclusively negative, participants in

410 the present study were able to identify some positive consequences of their hearing loss.

411 Previous studies investigating the positive aspects of acquired hearing loss have identified

412 major themes including reduced disturbance by unwanted sounds (Stephens \& Kerr, 2003),

413 recognition of one's ability to develop successful communication strategies (Manchaiah et al,

414 2015), and benefits of receiving communicative support from others to offload some of the

415 burden that hearing loss imposes (Lockey et al, 2010). These themes capture the positive

416 consequences identified by participants in the present study that included improvements in 
417 the ability to fall and stay asleep, the ability to develop coping strategies in the absence of

418 advice from clinicians, and the receipt of help from others.

420 The extent of the negative psychological and social consequences of SSD may perhaps be 421 surprising given the fact that these individuals have access to one largely unimpaired ear.

422 However, this apparent incongruence is compatible with the notion that it is not

423 straightforward to predict an individual's 'hearing handicap' ${ }^{1}$ based upon the level (as

424 measured by the audiogram) or lateralisation of hearing loss alone (Hallberg et al, 2008). A

425 hearing loss that is confined to one ear only cannot therefore be assumed to have only

426 minimal effects on health and well-being (Wie et al, 2010), which may be influenced strongly

427 by factors beyond the audiogram such as whether the onset of a hearing loss is gradual or 428 sudden (Sano, 2013). In fact, some aspects of the psychological and social consequences of 429 SSD were similar to those reported by individuals with mild and moderate levels of hearing 430 loss (Heffernan et al, 2016) and those with severe-to-profound losses (Mäki-Torkko et al, 431 2015). For example, individuals with hearing loss of varying degrees and laterality consistently report withdrawing from and within busy social situations, feeling negative emotions as a result of their hearing loss, and experiencing participation restrictions that have

434 a tangible impact on their everyday life. The burden of having to rely on other people to 435 communicate effectively was reported by the current participants but it has also been noted in 436 individuals with milder forms of hearing loss (Lockey et al, 2010). However, the present study also suggests that the burden imposed by SSD is highly situation specific whereby an individual's capacity to integrate and participate in a given situation may be altered by even a

\footnotetext{
${ }^{1}$ In this context, hearing handicap refers to the extent to which their hearing loss affects their behavior and wellbeing (Ventry \& Weinstein, 1982).
} 
small change in the listening environment. For example, participants reported having little or

440 no difficulty conversing with one person in a quiet room but also reported that they would

441 experience considerable difficulty with the addition of even low levels of background noise

442 or if even one other person spoke at the same time.

444 Although the CROS aid is the standard audiological intervention for those with SSD in the

445 UK where this study was conducted (Baguley et al, 2006; Kitterick et al, 2014), only one

446 participant in this study reported using one consistently but only in a specific listening

447 situation (watching television) and reported little or no benefit from doing so. The uptake and

448 use of CROS aids has been suggested to relate to the motivation of the patient to take up

449 technological support (Harford and Barry, 1965) and also to the cosmetic appearance and

450 discomfort from wearing an aid that may occlude, or be perceived to occlude, the normal-

451 hearing ear (Ryu et al, 2015). Uptake and usage may also be affected by a mismatch between

452 patient needs and device capabilities. However, approximately half of the participants had

453 never been offered a CROS aid and some of those individuals were completely unaware of

454 the intervention, suggesting that provision of CROS devices may also vary geographically in

455 the UK. This apparent lack of provision and uptake of hearing-assistive devices (e.g. CROS,

456 BCHD and CI) is particularly problematic in light of existing evidence for the beneficial

457 effects that they can have on quality of life by alleviating listening difficulties (Desmet et al,

458 2012; Hol et al, 2010; Kompis et al, 2017; Mertens et al, 2015; Ryu et al, 2015; Saliba et

459 al, 2011; Schrøder et al, 2010; Wesarg et al, 2013; Távora-Vieira et al, 2015) and increasing

460 overall health and well-being (Arndt et al, 2011). 
462 The fact that the burden imposed by SSD is not immediately obvious from the audiogram

463 alone and is not likely to be apparent in the quiet confines of the clinic may explain, at least

464 in part, why no participant in the present sample reported being referred for counselling or

465 offered non-technological forms of support or why so few appeared to have been offered

466 devices such as CROS aids. In particular, participants suggested that they would have

467 benefitted from receiving information about the long-term implications of their condition, the

468 potential benefits and limitations of available interventions and devices (e.g. CROS, BCHD

469 and CI), and advice on techniques and strategies for self-management. Participants also

470 reported that their coping strategies were a result of self-learning developed from years of

471 experience of living with their hearing loss rather than based on advice from a clinical

472 professional. It would therefore seem appropriate for the clinical management of individuals

473 with SSD to incorporate information-giving (Fig. 1, shaded decision nodes) to target the

474 counterproductive strategies that were identified by participants in the present study; i.e. the

475 sub-themes of withdrawing within situations (where the individual was motivated to attend

476 despite their listening difficulties) and withdrawing from situations (where participation was

477 considered counterproductive to their well-being). The provision of this information could

478 provide an opportunity to discuss the chronic symptoms and implications of the condition

479 ('lack of formal information' sub-theme), reduce any negative emotions associated with

480 engaging in social situations ('social interactions' sub-theme), and aim to develop motivation

481 for engagement and uptake of hearing-assistive devices such as CROS aids or BCHDs

482 ('hearing-assistive devices' sub-theme; Ferguson et al, 2016) (Fig. 1, 'Motivated to

483 participate?'). It could support individuals to develop realistic expectations about their ability

484 to integrate and participate with others ('social stigma' and 'self-stigma' sub-themes),

485 encourage positive beliefs around their ability to participate ('low self-efficacy' sub-theme;

486 Bandura, 1977), and ensure that individuals understand what benefits their hearing-assistive 
487 devices can provide and in what situations ('hearing-assistive devices' sub-theme; Fig. 1,

488 'Appropriate expectations?'). Finally, clinicians could deliver advice on developing positive

489 coping strategies such as how to position oneself within a social situation to maximise

490 audibility ('positioning in a social setting' sub-theme; Fig. 1, 'Aware of coping strategies?').

491 However, this advice would have to be modified to account for the use of rerouting devices

492 such as CROS aids and BCHDs; their use may conflict with listening strategies that would

493 otherwise be beneficial such as positioning unwanted noises toward the deaf ear.

494

495 The present qualitative analysis leads to the conclusion that SSD imposes a substantial degree

496 of burden across multiple domains of health that can lead to negative effects on psychological

497 well-being and restrictions on social participation. Almost 50 years ago, Giolas \& Wark

498 (1967) proposed that the clinical management of individuals with SSD should address these

499 negative effects and there is now a large body of evidence for how hearing-assistive devices

500 can alleviate listening difficulties and support participation in everyday life (Finke et al,

$5012017 b$; Kitterick et al, 2016; Peters et al, 2015). However, patients still identify a lack of

502 clinical support, which the present qualitative analysis suggests could be addressed through

503 information giving. The integration of this additional support into their clinical management

504 plan could help these individuals to develop, manage, and adopt effective coping strategies

505 and maximize take-up and use of hearing-assistive devices. 
506

507

508

509

510

511

512

513

514

515

516

517

518

519

520

521

522

523

524

525

526

527

528

529

\section{References}

Arndt, S., Aschendorff, A., Laszig, R., Beck, R., Schild, C., et al. (2011). Comparison of pseudobinaural hearing to real binaural hearing rehabilitation after cochlear implantation in patients with unilateral deafness and tinnitus. Otol neurotol, 32(1), 3947.

Arndt, S., Laszig, R., Aschendorff, A. et al. (2017). Cochlear implant treatment of patients with single-sided deafness or asymmetric hearing loss. HNO, 65(Suppl. 2), 98-108

Baguley, D. M., Bird, J., Humphriss, R.L., \& Prevost, A. T. (2006). The evidence base for the application of contralateral bone anchored hearing aids in acquired unilateral sensorineural hearing loss in adults. Clin Otolaryngol, 31, 6-14.

Bandura, A. (1977). Self-efficacy: Toward a Unifying Theory of Behavioral Change. Psych Rev, 84(2), 191-215.

Braun V. \& Clarke V. (2006). Using thematic analysis in psychology. Qual Res Psychol, 3, $77-101$

British Society of Audiology (2010). Recommended procedure: Ear examination. British Society of Audiology. Retrieved from http://bit.ly/bsarpearexam on 20th May 2016.

British Society of Audiology (2012). Recommended procedure: Pure-tone air-conduction and bone-conduction threshold audiometry with and without masking. British Society of Audiology. Retrieved from http://bit.ly/bsarppta on 20th May 2016.

Byl, F. M. Jr. (1984). Sudden hearing loss: eight years experience and suggested prognostic table. Laryngoscope, 94, 647-661.

Busk Linnebjerg, L., Wetke, R. (2014). The benefits of CROS aids for individuals with unilateral sensorineural hearing loss. Hearing Balance Commun, 12(1), 36-40. 
530 Choissoine-Kerdel, J. A., Baguley, D. M., Stoddart, R. L, Moffat, D. A. (2000). An

531 investigation of the audiologic handicap associated with unilateral sudden 532 sensorineural hearing loss. Am J Otol, 21, 645-651.

533 Cox, R. M., \& Alexander, G. C. (1995). The abbreviated profile of hearing aid benefit. $534 \quad$ Ear hear, 16(2), 176-186.

535 Davis, A. (1995). Hearing in Adults. London: Whurr.

536 Desmet, J. B. J., Wouters, K., De Bodt, M., Van de Heyning, P. (2012). Comparison of 2 537 implantable bone conduction devices in patients with single-sided deafness using a 538 daily alternating method. Otol Neurotol, 33(6), 1018-1026.

539 Douglas, S. A., Yeung, P., Daudia, A., Gatehouse, S., O’Donoghue, G. M. (2007).

$540 \quad$ Spatial hearing disability after acoustic neuroma removal. Laryngoscope, 117, $541 \quad 1648-1651$.

542 Dwyer, N. Y., Firszt, J. B., Reeder, R. M. (2014). Effects of unilateral input and mode of 543 hearing in the better ear: self-reported performance using the speech, spatial and 544 qualities of hearing scale. Ear Hear, 35(1), 126-136.

545 Ferguson, M., Maidment, D., Russell, N., Gregory, M., Nicholson, R. (2016).

546 Motivational engagement in first-time hearing aid users: a feasibility study. Int $J$ $547 \quad$ Audiol, 55(Suppl. 3), S23-33.

548 Finbow, J., Bance, M., Aiken, S., Gulliver, M., Verge, J., et al. (2015). A comparison 549 between wireless CROS and bone-anchored hearing devices for single-sided 550 deafness: a pilot study. Otol Neurotol, 36(5), 819-825.

551 Finke, M., Strauß-Schier, A., Kludt, E., Büchner, A., Illg, A. (2017a). Speech 552 intelligibility and subjective benefit in single-sided deaf adults after cochlear implantation. Hearing res, 348, 112-119. 
554 Finke, M., Bönitz, M., Lyxell, B. et al (2017b) Cochlear implant effectiveness in postlingual single-sided deaf individuals: what's the point?, Int J Audiol, 56(6), 417-423, DOI: $10.1080 / 14992027.2017 .1296595$

Flanagan, J. C. (1954). The critical incident technique. Psychol Bull, 51, 327.

Fusch, P. I. \& Ness, L. R. (2015). Are we there yet? Data saturation in qualitative research. The Qualitative Report. 20(9), 1408-1416.

Gatehouse, S., \& Noble, W. (2004). The speech, spatial and qualities of hearing scale (SSQ). Int J Audiol, 43(2), 85-99.

Giolas, T. G. \& Wark, D. J. (1967). Communication problems associated with unilateral hearing loss. J Speech Hear Disord, 32, 336-343.

Glaser, B. G. \& Strauss, A. L. (1967). The discovery of grounded theory: strategies for qualitative research. New York: Aldine Publishing Company.

Hallberg, L. R. M., Hallberg, U., Kramer, S. E. (2008). Self-reported hearing difficulties, communication strategies and psychological general well-being (quality of life) in patients with acquired hearing impairment. Disabil Rehabil, 30(3), 203-212.

Harford, E. \& Barry, J. (1965). A rehabilitative approach to the problem of unilateral hearing impairment: The contralateral routing of signals (CROS). J Speech Hear Disord, 30, 121-138.

Härkönen, K., Kivekäs, I., Rautiainen, M., Kotti, V., Sivonen, V., et al. (2015). Singlesided deafness: the effect of cochlear implantation on quality of life, quality of hearing, and working performance. ORL, 77(6), 339-345.

Hassepass, F., Arndt, S., Aschendorff, A., Laszig, R., Wesarg, T. (2016). Cochlear implantation for hearing rehabilitation in single-sided deafness after translabyrinthine vestibular schwannoma surgery. Eur Arch Otorhinolaryngol, 273(9), 2373-2383. 
579 Hawley, M. L., Litovsky, R. Y., Culling, J. F. (2004). The benefit of binaural hearing in 580 a cocktail party: effect of location and type of interferer. J Acoust Soc Am, 115, $833-843$.

Heffernan, E., Coulson, N. S., Henshaw, H., Barry, J. G., Ferguson, M. A. (2016). Understanding the psychosocial experiences of adults with mild-moderate hearing loss: an application of Leventhal's self-regulatory model. Int J Audiol, 55 (Suppl. 3), S3-S12.

Hol, M. K., Kunst, S. J., Snik, A. F., Cremers, C. W. (2010). Pilot study on the effectiveness of the conventional CROS, the transcranial CROS and the BAHA transcranial CROS in adults with unilateral inner ear deafness. Eur Arch Otorhinolaryngol, 267(6), 889-896.

Iwasaki, S., Sano, H., Nishio, S., Takumi, Y., Okamoto, M., et al. (2013). Hearing handicap in adults with unilateral deafness and bilateral hearing loss. Otol Neurotol, 34(4), 644-649.

Jacob, R., Stelzig, Y., Nopp, P., Schleich, P. (2011). Audiological results with cochlear implants for single-sided deafness. $H N O, 59(5), 453-460$.

Kitterick, P. T., O’Donoghue, G. M., Edmondson-Jones, M., Marshall, A., Jeffs, E., et al. (2014). Comparison of the benefits of cochlear implantation versus contra-lateral routing of signal hearing aids in adult patients with single-sided deafness: study protocol for a prospective within-subject longitudinal trial. BMC Ear Nose Throat Dis,

600 Kitterick, P. T., Lucas, L., \& Smith, S. N. (2015). Improving health-related quality of life in 601 single-sided deafness: a systematic review and meta-analysis. Audiol Neurotol, 20(Suppl. 1), 79-86. 
603 Kitterick, P. T., Smith, S. N., Lucas, L. (2016). Hearing-assistive devices for unilateral severe-to-profound sensorineural hearing loss in adults: A systematic review and metaanalysis. Ear Hear, 37(5), 495-507.

Kompis, M., Wimmer, W., Caversaccio, M. (2017). Long term benefit of bone anchored hearing systems in single sided deafness. Acta Oto-laryngol, 137(4), 398-402.

Lin, L. M., Bowditch, S., Anderson, M. J., May, B., Cox, K. M., et al. (2006). Amplification in the rehabilitation of unilateral deafness: speech in noise and directional hearing effects with bone-anchored hearing and contralateral routing of signal amplification. Otol Neurotol, 27(2), 172-182.

Lockey, K., Jennings, M. B., Shaw, L. (2010). Exploring hearing aid use in older women through narratives. Int J Audiol, 49(8), 542-549.

Manchaiah, V., Baguley, D. M., Pyykkö, I., Kentala, E., Levo, H. (2015). Positive experiences associated with acquired hearing loss, Ménière's disease, and tinnitus: a review. Int J Audiol, 54(1), 1-10.

Mäki-Torkko, E. M., Vestergren, S., Harder, H., Lyxell, B. (2015). From isolation and dependence to autonomy-expectations before and experiences after cochlear implantation in adult cochlear implant users and their significant others. Disabil Rehabil, 37(6), 541-547.

McColl, E., Jacoby, A., Thomas, L., Soutter, J., Bamford, C., et al. (2001). Design and use of questionnaires: a review of best practice applicable to surveys of health service staff and patients. Health Technol Assess, 5(31), 1-256.

McLeod, B., Upfold, L., Taylor, A. (2008). Self reported hearing difficulties following excision of vestibular schwannoma. Int J Aud, 47, 420-430. 
626 Mertens, G., Kleine Punte, A., De Bodt, M., Van de Heyning, P. (2015). Binaural auditory outcomes in patients with postlingual profound unilateral hearing loss: 3 years after cochlear implantation. Audiol Neurotol, 20(Suppl. 1), 67-72.

Newman, C. W., Jacobson, G. P., Hug, G. A., Sandridge, S. A. (1997). Perceived hearing handicap of patients with unilateral or mild hearing loss. Ann Otol Thinol Laryngol, $106,210-214$.

Newman, C. W., Sandridge, S. A., Oswald, L. M. (2010). Relationship between expectations and satisfaction for Baha implant system in patients with single-sided deafness. Semin Hear, 31(1), 15-27.

Niparko, J. K., Cox, K. M., Lustig, L. R. (2003). Comparison of the bone anchored hearing aid implantable hearing device with contralateral routing of offside signal amplification in the rehabilitation of unilateral deafness. Otol Neurotol, 24(1), 73-

NVivo qualitative data analysis software; QSR International Pty Ltd. Version 10, 2012

O'Cathain, A. \& Thomas, K. J. (2004). "Any other comments?" Open questions on questionnaires-a bane or a bonus to research? BMC Med Res Methodol, 4(1), 25.

Peters, J. P., Smit, A. L., Stegeman, I., Grolman, W. (2015). Review: Bone conduction devices and contralateral routing of sound systems in single-sided deafness. Laryngoscope, 125, 218-226.

Ramos, M. A., Falcon Gonzalez, J.C., Manrique, M., Morera, C., García-Ibáñez, L., et al. (2015). Cochlear implants as a treatment option for unilateral hearing loss, severe tinnitus and hyperacusis. Audiol Neurotol, 20(Suppl. 1), 60-66. 
651 Saliba, I., Nader, M. E., El Fata, F., Leroux, T. (2011). Bone anchored hearing aid in

652

653

654

655

656

657

658

659

660

661

662

663

664

665

666

667

668

669

670

671

672

673 single sided deafness: Outcome in right-handed patients. Auris Nasus Larynx, 38(5), 570-576.

Sano, H., Okamoto, M., Ohhashi, K., Ino, T., Iwasaki, S., et al. (2013). Self-reported symptoms in patients with idiopathic sudden sensorineural hearing loss. Otol Neurotol, 34, 1405-1410.

Schrøder, S. A., Ravn, T., Bonding, P. (2010). BAHA in single-sided deafness: patient compliance and subjective benefit. Otol Neurotol, 31(3), 404-408.

Stephens, D. \& Kerr, P.C. (2003). The role of positive experiences in living with acquired hearing loss. Int J Audiol, 42 (S1), 118-127.

Subramanium, K., Eikelboom, R. H., Eager, K. M., et al. (2005). Unilateral profound hearing loss and the effect on quality of life after cerebellopontine angle surgery. Otolaryngol - Head Neck Sur, 113, 339-346.

Távora-Vieira, D., Marino, R., Acharya, A., Rajan, G. P. (2015). The impact of cochlear implantation on speech understanding, subjective hearing performance, and tinnitus perception in patients with unilateral severe to profound hearing loss. Otol Neurotol, 36(3), 430-436.

Valente, M., Barninger, K. H., Oeding, K., Smith, S., Snapp, H., Sydlowski, S. (2015). Clinical Practice Guidelines: Adult Patients with Severe-to-Profound Unilateral Sensorineural Hearing Loss. American Academy of Audiology.

Van de Heyning, P., Vermeire, K., Diebl, M., Nopp, P., Anderson, I., De Ridder, D. (2008). Incapacitating unilateral tinnitus in single-sided deafness treated by cochlear implantation. Ann Otol Rhinol Laryngol, 117(9), 645-652. 
674 Vermeire, K., \& Van de Heyning, P. (2009). Binaural hearing after cochlear implantation in 675 subjects with unilateral sensorineural deafness and tinnitus. Audiol Neurotol, 14(3), $676 \quad 163-171$.

677 Vincent, C., Arndt, S., Firszt, J. B., et al. (2015). Identification and Evaluation of 678 Cochlear Implant Candidates with Asymmetrical Hearing Loss. Audiol Neurotol, $679 \quad 20($ Suppl 1), 87-89.

680 Welsh, L. W., Welso, J. J., Rosen, L.F., et al. (2004). Functional impairments due to 681 unilateral deafness. Ann Otol Rhinol Laryngol, 113(12), 987-993.

682 Wesarg, T., Aschendorff, A., Laszig, R., Beck, R., Schild, C., Hassepass, F., Kroeger, S., 683 Hocke, T., Arndt, S. (2013). Comparison of speech discrimination in noise and 684 directional hearing with 2 different sound processors of a bone-anchored hearing 685 system in adults with unilateral severe or profound sensorineural hearing loss. Otol $686 \quad$ Neurotol, 34(6), 1064-1070.

687 Wie, O. B., Pripp, A. H., Tvete, O. (2010). Unilateral deafness in adults: Effects on 688 communication and social interaction. Ann Otol, Rhinol Laryngol, 119, 772-781. 


\section{Figure captions}

690 Figure 1: Flow chart demonstrating the main coping strategies that participants reported

691 using in social situations and the sequence of events that prompt the use of a given

692 strategy; i.e. positive engagement, withdrawal from situations (social isolation /

693 avoidance), or withdrawal within situations (introversion). Events are indicated using

694 rectangles and decisions are indicated using diamonds. The potential influences of

695 counselling on behavior are indicated by the shaded decision nodes. 


\section{Figure 1}

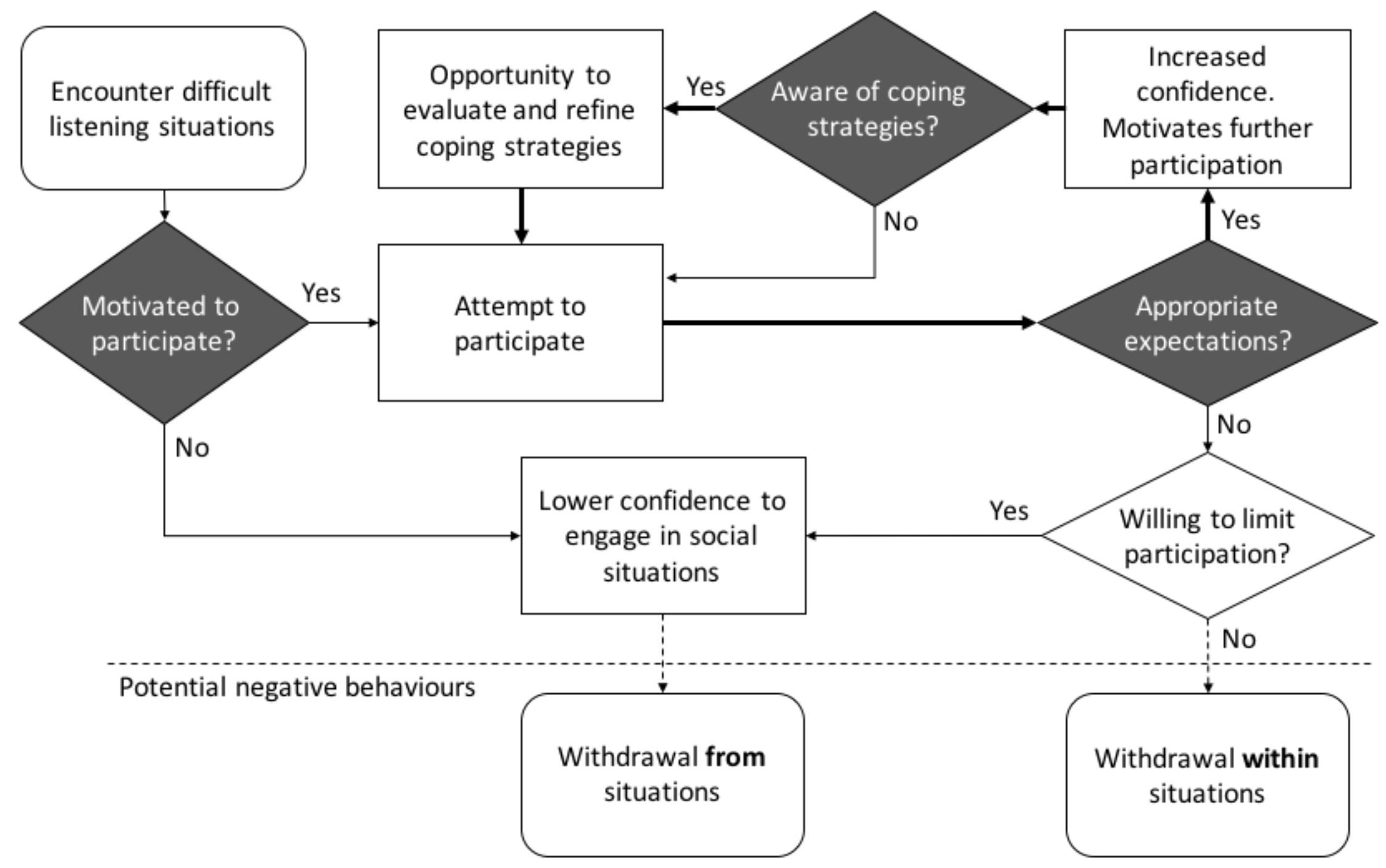


Table 1: Demographic information for the individuals with single-sided deafness that participated in the interviews.

\begin{tabular}{|c|c|c|c|c|c|c|c|c|}
\hline $\begin{array}{r}\text { Participant } \\
\text { ID }\end{array}$ & Gender & Age & Employment status & Onset & Aetiology & Duration & Tinnitus & $\begin{array}{l}\text { Reported } \\
\text { device use }\end{array}$ \\
\hline P1 & female & 67 & retired & sudden & idiopathic & $23+$ years & yes & Hearing aid \\
\hline $\mathbf{P 2}$ & male & 71 & retired & sudden & surgery for & 8 years, 5 months & yes & CROS \\
\hline & & & & & vestibular schwannoma & & & \\
\hline P3 & female & 53 & self-employed part time & progressive & meningitis & 26 years, 9 months & yes & None \\
\hline $\mathbf{P 4}$ & female & 68 & retired & sudden & idiopathic & 5 years & no & None \\
\hline P5 & male & 66 & retired & progressive & age-related decline & 28 years & yes & None \\
\hline P6 & female & 56 & employed full-time & sudden & idiopathic & 2 years & yes & None \\
\hline P7 & male & 40 & employed full-time & sudden & surgery for & 6 years, 4 months & yes & None \\
\hline & & & & & vestibular schwannoma & & & \\
\hline P8 & male & 37 & employed full-time & sudden & idiopathic & 1 year, 1 month & yes & None \\
\hline
\end{tabular}




\begin{tabular}{|c|c|}
\hline Activity & Description \\
\hline Introduction & $\begin{array}{l}\text { The facilitator gave an overview of the research question, the motivation for asking the question, and presented the structure } \\
\text { of the activity. Participants were able to ask questions before proceeding on to the first activity. }\end{array}$ \\
\hline $\begin{array}{l}\text { Topic } \\
\text { generation }\end{array}$ & $\begin{array}{l}\text { Participants were provided with red and green 'topic' cards and asked to fill in the cards without conferring with others. The } \\
\text { red (or green) cards captured situations in which their hearing loss created (or did not create) difficulties for them or had } \\
\text { negative (or positive) consequences. To ensure that the topic generation exercise captured all relevant issues, no matter how } \\
\text { sensitive, each card included a box that participants could tick to indicate that they did not wish the topic to be discussed with } \\
\text { the other participants. }\end{array}$ \\
\hline Break & $\begin{array}{l}\text { The facilitator and co-facilitator grouped cards that contained similar topics for discussion (e.g. localising sounds, } \\
\text { communication, social impacts, psychological effects, etc.). }\end{array}$ \\
\hline $\begin{array}{l}\text { Semi-structured } \\
\text { discussion }\end{array}$ & $\begin{array}{l}\text { Participants were handed back their responses cards. The facilitator read out each identified topic in turn and proceeded to } \\
\text { ask questions to seek clarification or to gain a better understanding of the situation or consequences linked to each topic. This } \\
\text { process was repeated until all topics and responses had been discussed. The facilitator encouraged all participants to } \\
\text { contribute to each topic of discussion to ensure all views were captured. The semi-structured discussion ended when the } \\
\text { participants felt that all topics written on their cards had been discussed. }\end{array}$ \\
\hline $\begin{array}{l}\text { Unstructured } \\
\text { discussion }\end{array}$ & $\begin{array}{l}\text { An open-ended question was posed by the facilitator to capture any other situations or consequences related to their hearing } \\
\text { loss that had not yet been discussed. Any additional topics were discussed as in the semi-structured discussion. }\end{array}$ \\
\hline Debriefing & $\begin{array}{l}\text { Participants were thanked for their involvement, reminded of the purposes of the study, and informed that the results would } \\
\text { be provided to them when available. Before leaving, participants were provided with an information sheet containing the } \\
\text { contact details of national charities and support organisations related to hearing loss and well-being and were informed to } \\
\text { contact their family doctor with any concerns about their hearing, health or well-being. }\end{array}$ \\
\hline
\end{tabular}

Table 2: Sequence and description of activities in each of the three interviews. 
Table 3: Examples of similar topics identified by patients in the 'topic generation exercise' across the three group interviews.

\begin{tabular}{l|l|l}
\hline Interview 1 & Interview 2 & Interview 3 \\
\hline $\begin{array}{l}\text { P3: "Group discussion in a room } \\
\text { with background noise e.g. } \\
\text { music, TV or other conversation } \\
\text { going on." }\end{array}$ & $\begin{array}{l}\text { P2: "Group conversations, particularly in } \\
\text { social settings where people, } \\
\text { understandably, talk over each other." }\end{array}$ & $\begin{array}{l}\text { P7: "Group conversations i.e. when there is } \\
\text { background noise." }\end{array}$ \\
\hline $\begin{array}{l}\text { P5: "Need to position myself on } \\
\text { the corner of a table in order to } \\
\text { hear as much as possible with my } \\
\text { good ear." }\end{array}$ & P2: "Concerts - need to choose sides." & $\begin{array}{l}\text { P4: "Joining a meeting late and not being able } \\
\text { to select a seat which aids my SSD." }\end{array}$ \\
$\begin{array}{l}\text { P5: "I have no appreciation of } \\
\text { music in stereo. This is very } \\
\text { noticeable when I recollect exactly } \\
\text { what a particular piece of music or } \\
\begin{array}{l}\text { a play with sound effects was like } \\
\text { when I could hear with both ears." }\end{array}\end{array}$ & P6: "Music - I miss stereo sound." & P8: "I miss stereo music with headphones." \\
$\begin{array}{l}\text { P5: "Knowing which direction } \\
\text { traffic is approaching from." }\end{array}$ & $\begin{array}{l}\text { P6: "Direction - If I'm walking across a } \\
\text { road I have to rely on my eyes to know } \\
\text { where a car is coming from." }\end{array}$ & $\begin{array}{l}\text { P7: "Can't tell which direction sound is } \\
\text { coming from." }\end{array}$ \\
\hline
\end{tabular}




\section{List of Supplemental Digital Content}

Supplemental Digital Content 1: Table listing the individual topics identified by

participants themselves using the Critical Incident Technique within each category. Pdf 
The raw unedited text from the topic cards completed by participants during the 'topic generation exercise'. Similar negative and positive topics have been grouped together within each interview.

\section{Interview 1: Negative consequences / difficulties \\ Concern over loss of hearing in good ear.}

Fear of anything happening to the other 'good' ear.

Feeling isolated, frustrated and left out of conversations - only hear snippets of what is said and lack of confidence to join in. Feel like people think you are dull or have nothing to say.

If someone approaches on my deaf side and I can't see them it makes me jump.

Knowing which direction traffic is approaching from.

Discerning direction of sound. E.g. traffic noise or of who is speaking where.

Having to go around in circles to ascertain the direction of a noise.

Unable to tell where family members are in the house if they are not in the same room as me. My first question is always 'where are you?' 'I'm here' does not help.

Need to position myself on the corner of a table in order to hear as much as possible with my good ear.

As my left ear is profoundly deaf, listening to the radio in a car is harder on the passenger side than on the driver side. Also hearing a person whilst driving is difficult.

Other people not being sympathetic. People shouting when I tell them I have a hearing loss or don't bother trying to talk to you. Lack of willingness to engage. Other people exasperated because you can't hear.

Networking situations at work, particularly with people who don't know me and don't understand about my hearing disability.

People will repeat something once, but do not want to repeat it again if asked. Eventually they talk to someone else because it is less of an effort for them.

If I'm at a table at a formal or informal dinner I have to notify the person on my left that if they say something I won't hear them. This usually ensures that they do not make any effort to talk to me.

Trying to get workplace to make reasonable adjustments and having to fight. Positioning myself at work in open plan office - need to be in a corner with colleagues to right and in front of me. Convincing people of this and having to fight for the position is frustrating.

My ex-boss who used to say 'pardon?' every time I said I had a hearing loss (his little joke) until someone pointed out it was not funny.

Airports / train stations can't hear announcements.

Noisy young children - can't hear what other people are saying.

Group discussion in a room with background noise e.g. music, TV or other conversation going on.

One to one discussion in a noisy environment. E.g. noisy pub / restaurant. I can do this but it is tiring. Sometimes ask to move e.g. in corner or against wall.

Syndicate groups in e.g. a work conference if more than one group in the same room.

Certain social occasions e.g. meal in busy restaurant with lots of chatter - strain to follow conversation and person on deaf side. Effort to concentrate on them - fatigue.

If someone is on my left and actually makes an effort to speak to me I have to turn my ear and cup my right ear. This is very fatiguing on a long airplane flight.

I have no appreciation of music in stereo. This is very noticeable when I recollect exactly what a particular piece of music or a play with sound effects was like when I

could hear with both ears. 
I have continuous tinnitus in my left ear due to cochlear damage caused by an infection following a stapedotomy. Other SSD impaired people may have the same problem.

Check for cause of SSD if it is a problem with the cochlear they will probably suffer with tinnitus.

I have no balance mechanism due to my left ear. This could also apply to others whose loss of hearing is caused by cochlear damage.

\section{Interview 1: Positive consequences / no difficulty}

One to one discussion in quiet environment.

Group discussion in quiet room where only one person talks at once.

Group conversation in a quiet room is $\mathrm{OK}$.

Group discussion in a quiet room where only one person talks at once.

Sleeping - Sleep on good ear if there is noise.

Being in a nosy environment if I'm with someone e.g. a friend who understands.

Music.

Tinnitus doesn't bother me any more even though it's there all the time. I tune out from it.

TV usually OK

TV is ok as it is not usually in stereo.

Telephone usually OK.

Telephone is OK with hearing ear.

Telling people I have a hearing disability.

\section{Interview 2: Negative consequences / difficulties}

Direction - If I'm walking across a road I have to rely on my eyes to know where a car is coming from.

Direction - When I'm driving - where is the ambulance.

Direction - I can't tell where somebody is. If my husband says 'I'm here' I can't tell where he is. He has to say 'I'm in the kitchen'.

Direction - If I drop something I've no idea where it rolls to.

No directional information.

Frustration. Where is the squeak coming from in the car?

In the car. When I drive, my wife is on my deaf side.

Music - I miss stereo sound.

Sound 'topography' is 'flat' so bird song etc. is lacking (possibly a directional problem?)

Concerts - need to choose sides.

Have difficulty in noise (crowded) situations and find I just hear a general hubbub of noise and have to be very close to somebody to hear what they are saying.

At work - meetings are often difficult if too many people talk at once, or especially if there is a phone link to another global office. I have to make sure I sit close to the speaker.

Group conversations, particularly in social settings where people, understandably, talk over each other. 
I work in an open-plan environment and find it difficult to hear some people (especially those who speak quietly). I have to go into a quiet room to phone somebody otherwise I have difficulty distinguishing their voice over the voice of others.

Confusing 'live' sound with that which is on the TV \& radio.

Telephone - I want to hold the phone in my right hand, but I often want to jot down a note and I'm right handed.

Telephone - Can't switch ears. If my arm gets tired it's difficult. In a public situation if somebody wants to get past me and they talk into my left (bad) ear saying 'excuse me' they often think I'm rude and ignoring them.

I'm more antisocial, as I find conversations more difficult. It seems that 'muzak' is more often found in restaurants, waiting rooms etc.

Withdrawal of my active interest. Previously I might seek the sources of an unexpected sound, now, less often.

I use a 'wake \& shake' alarm clock, because I sleep mostly on my good ear. However, I worry about not hearing other sounds at night - like my burglar alarm going off.

I find I'm saying 'excuse me' a lot of the time and people have to repeat what they say.

Cycling - balance.

Tinnitus possibly worse, very one-sided.

\section{Interview 2: Positive consequences / no difficulty}

I've found I can lip read better than most people.

I've realised how good the hearing is in my right ear and there are millions of worse things a person can have.

I can switch of noise more easily sometimes at work when I'm concentrating.

Sleeping - I can put hearing ear into the pillow.

Somehow, I hear things I'm not intended to hear. Change in tone on speaking 'sotto voce'

\section{Interview 3: Negative consequences / difficulties}

Background noise - find it hard to hear as just one ear trying to deal with all the 360 degree sound.

Completely ignore people on my deaf side if they speak and there is any background noise.

Parties are a nightmare (but sometimes I am quite glad of an excuse to opt out).

Group conversations i.e. when there is background noise.

Very difficult to interact in groups larger than 2-3 people.

Conversations within a group socially.

Had difficulty with meetings at work.

If more than one person talks at once I give up and have to tell whoever is speaking to wait.

One to one conversations on 'deaf side' in a noisy environment.

Speaking to my son, when I pick him up from school (he's 7), so therefore small, with background noise I can't hear what he is saying.

Talking on the phone if there is background noise.

Telephone- have to use my other ear now.

Had difficulty taking telephone calls at work. 
Listening whilst on the phone.

Talking on the phone for a long period of time as arm aches and can't swap ears.

Hearing bicycles / people behind me when out walking.

Hearing people who are talking behind me.

Again - not knowing where sounds are coming from, crossing the road takes much more concentration, is more dangerous.

No idea where sounds are coming from - someone calls me, I have no idea where to look. The mobile phone goes and I don't know where to go to answer it.

Can't tell which direction sound is coming from.

Can be slightly concerning when cycling as can't always hear where cars are coming from.

Can't hear when I am a passenger in a car, and I have to twist my head around.

Have to continually turn my head to one side to listen to noises/ conversation in front of me. Can result in a sore neck.

Constantly have to change the side I walk when walking/talking to people even after continuously telling people I need to be on their right they don't listen.

I always position myself on the outside of a group as it is impossible to be in the middle and try and keep up with what's going on.

Finding good places to sit when eating with friends.

Joining a meeting late and not being able to select a seat which aids my SSD.

It can be tiresome having to tell total strangers why you don't hear them or why they have to talk on my good side. In restaurants / on trains/ on planes.

A lot of the time I can phase out thinking about the problems but sometimes it feels impossible that I must be like this for the rest of my life.

Periodic depression.

A feeling that no-one understands and that they would only take any interest if I was deaf completely.

Difficult hearing shop assistants - I feel stupid.

Sometimes family forget and I get frustrated when I can't hear - it feels very unfair.

Getting people to understand that I can't hear on the left side so please talk to me on my right.

I don't feel part of large gatherings and no longer am able to enjoy large socials.

I always avoid large groups and my socialising is almost non-existent because of this.

I hate to be thought of as rude for ignoring people on my SSD side who aren't aware.

Terror about any problem with my good ear.

The unknown 'SSD' causes, etc. No answers from medical professionals, sometimes dismissive, left in the dark.

Questions' about further recurrence, further deafness.

Will my hearing deteriorate in the right ear?

Enjoyment of music - now only in mono, some distortion at certain levels.

I miss stereo music with headphones.

Distorted hearing at certain volumes.

I find it exhausting listening to someone when there is background noise.

I get much more tired from the extra effort it takes to concentrate and spend more time lying down. 
Tinnitus - since hearing loss night and day - awful.

Am aware that I have some tinnitus in both ears, but with the one with no hearing it is intense and sometimes makes me feel desperate.

I can feel very dizzy if walking in crowds and noisy environments (possibly not as a result of SSD but because of acoustic neuroma surgery).

I have some dizziness left over from labrynthitis, but I am not sure if this is made worse by the deafness - affecting my balance.

Interview 3: Positive consequences / no difficulty

Not any real problem watching TV if it is just me in the room and no other noise.

Ear plugs to cancel out noise last twice as long.

If trying to sleep when there is noise I can lay on one side and not hear the surrounding noise.

I'm not kept awake when the neighbours have parties.

I was only the only one in the house who slept well when my little granddaughter was born.

I can't hear my husband snoring.

Better with one-to-one conversations. 